\title{
Reversing radial segregation and suppressing morphological instability during vertical Bridgman crystal growth by rotation
}

\author{
C.W. Lan*, Y.W. Yang, C.Y. Tu \\ Department of Chemical Engineering, National Taiwan University, Taipei, Taiwan 10617, ROC
}

Received 26 September 2001; accepted 2 November 2001

Communicated by Dr. D.T.J. Hurle

\begin{abstract}
During vertical Bridgman growth of an alloy crystal, local accumulation of solute at the interface center due to buoyancy often causes formation of a deep depression or pit and accelerates morphological instability. Through visualization of the freezing interface during directional solidification of succinonitrile containing ethanol on a rotating table, it was shown that rotation about the ampoule axis might reverse solute distribution and remove pit formation. Simulation was also conducted to explain the observations. (C) 2002 Elsevier Science B.V. All rights reserved.
\end{abstract}

PACS: 44.25. +f; 47.27.Te; 81.10.Fq; 02.60.c6; 02.70.Fj

Keywords: A1. Centrifugal force; A1. Convection; A1. Interface; A1. Rotation; A1. Segregation; A2. Bridgman method

\section{Introduction}

The control of segregation and interface morphology is important in crystal growth. The classic constitutional supercooling [1,2] gives an upper stability limit of a planar interface. Exceeding this limit without convection, the interface may break down into cellular or dendritic structures. However, in reality, due to the presence of buoyant convection, the solute is not uniformly distributed at the interface. For a typical situation having a concave interface, caused by the release of heat of fusion or the smaller crystal thermal conductivity, the induced inward thermal convection causes local solute accumulation at the interface center.

\footnotetext{
*Corresponding author. Tel./fax: + 886-2-2363-3917.

E-mail address: lan@ruby.che.ntu.edu.tw (C.W. Lan).
}

For a non-dilute alloy, this often generates an interface depression or pit and further accelerates morphological breakdown. This instability usually occurs much earlier than the theoretical prediction $[3,4]$. To avoid the pit formation, the control of convection patterns and radial segregation near the growth front is thus important.

The use of external forces can be an effective way for control of convection. For example, magnetic fields have been widely adopted (e.g. [5]). Solidification or crystal growth in microgravity can also be helpful [6]. Recently, the use of a centrifuge $[7,8]$, i.e., the so-called centrifugal or high-gravity processing, has become popular and may open a new era to processing technology. However, in a recent numerical study by Lan and $\mathrm{Tu}$ [9], as well as some theoretical analysis by Skudarnov et al. [10], it was found that the "free- 
swing centrifuge configuration" might not be the best configuration for crystal growth. Lan and $\mathrm{Tu}$ [9] illustrated that the free-swing system generates 3D flows and severe solute non-uniformity. Surprisingly, they also found that rotation of a vertical ampoule is a better way for minimizing solute mixing and improving compositional uniformity (both radial and axial). Lan [11] further predicted that at a high rotation rate, the flow direction near the solidification front might even be reversed. If this concept is correct, the centrifuge can be replaced by a rotating table and the centrifugal processing can become much more compact and feasible in practice. Unfortunately, there has been no experiment to support this concept. In this paper, we report experimental results obtained by using a transparent system to prove this idea. The inversion of radial solute segregation by centrifugal acceleration was shown for the first time.

\section{Experiments and numerical simulation}

Succinonitrile ( $\mathrm{SCN}$ ) was directionally solidified using a transparent vertical Bridgman system. In addition to its transparency, SCN solidifies with a non-facetted interface like metallic materials and a high-purity sample can easily be prepared. In this study, SCN (Furuka Inc., about 99\% purity) was purified first by vacuum distillation at $50 \mathrm{mTorr}$ for ten times. The melting point of the purified sample was measured about $58.080^{\circ} \mathrm{C}$ by a triplepoint cell using a thermistor indicating that the purity of the sample is higher than $99.999 \%$ [12]. The distilled sample was collected in a $17-\mathrm{mm}$ diameter Pyrex ampoule ( $2.5 \mathrm{~mm}$ in thickness) and further purified by 16 passes through a three-zone refiner. The last $40 \%$ of the solidified sample was discarded. To perform the solidification experiments, about $0.005 \mathrm{wt} \%$ of ethanol was added into the sample through a $5 \mu \mathrm{l}$ microsyringe inserted into the bottom of the sample. The total sample length was $20 \mathrm{~cm}$.

The Bridgman furnace consisted of two heating zones made of copper blocks each with a Nichrome wire inside as a heating element. In between, a transparent insulation zone made of
Plexiglas was used. The hot- and cold-zone temperatures were controlled independently by two PID controllers and the temperatures were set to $80^{\circ} \mathrm{C}$ (top) and $40^{\circ} \mathrm{C}$ (bottom), respectively. The thermal gradient at the interface was measured by an immersed thermocouple traveling with the sample. By taking an average of the gradients at the interface from solidification and melting curves, we got the thermal gradient being about $8-10 \mathrm{~K} / \mathrm{cm}$. To translate the ampoule accurately, a microstepping motor was used to drive a screw slide; the translation rate was controlled at $2 \mu \mathrm{m} / \mathrm{s}$ in this study. During solidification, a video camera recorded the evolution of the interface morphology with a back lighting to enhance the contrast of the image. The whole solidification system was installed on a rotating table $(80 \mathrm{~cm}$ in diameter $)$, as shown in Fig. 1. The maximum vibration amplitude at the edge of the table surface was $<0.2 \mathrm{~mm}$ at $200 \mathrm{rpm}$, while the rotation speed was controlled within $1 \mathrm{rpm}$. The ampoule position was carefully adjusted by a $\mathrm{He} / \mathrm{Ne}$ laser beam during rotation, so that the rotation axis is aligned as much as possible with the ampoule axis.

An axisymmetric model accounting for melt convection, heat and mass transfer, and the moving interface $[4,13]$ was used to simulate the growth and better understand the observed phenomena. In brief, the model is based on a stream function/vorticity formulation and is solved by an efficient finite volume method. The heating profile is described by a hyperbolic tangent function fitting to the measured one; the thermal gradient at the interface ranging from 8 to $10 \mathrm{~K} / \mathrm{cm}$ is also used to best fit the observed results. The heat transfer coefficient $h$ is also a fitting parameter; $h=1.6 \times 10^{-2} \mathrm{~W} \mathrm{~cm}^{-2} \mathrm{~K}^{-1}$, which is the same as the one used in the previous calculations [4]. However, we find that this value is not very sensitive to the calculated results (unless using a very low value). Such an approximation is found to be adequate by comparing the one obtained by the Fluent code through a global simulation. Furthermore, to perform the dynamic simulation, the ampoule is set to be stationary, while the heating profile is moved upward at a given speed. The upper melt surface is set to be stress-free, while the no-slip boundary condition is adopted 


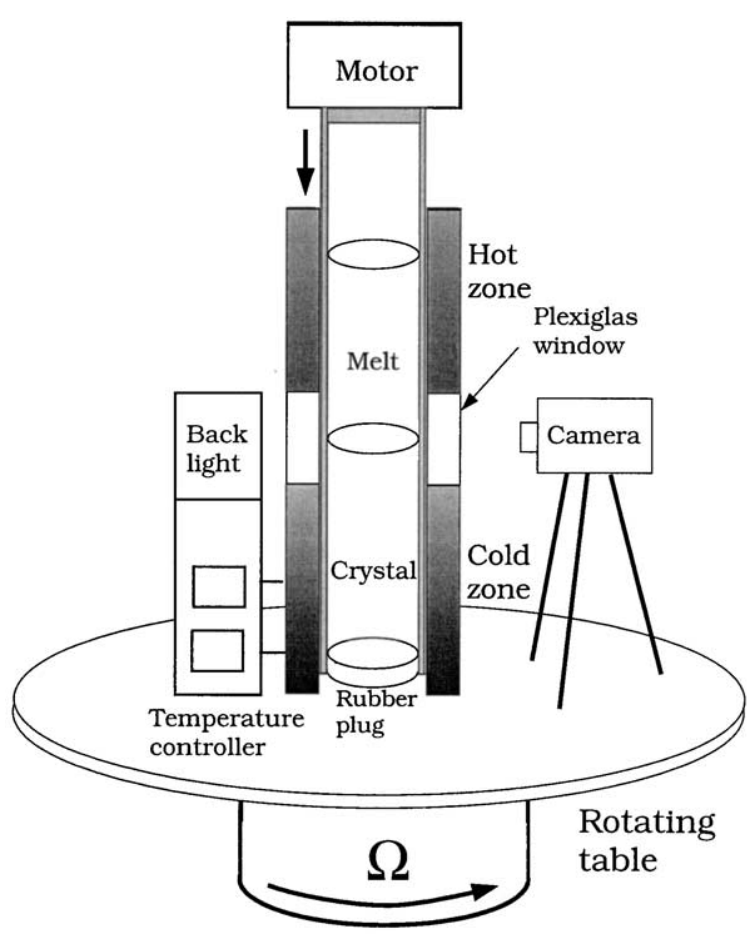

Fig. 1. Sketch of the experimental setup.

for all solid boundaries. More importantly, because ethanol absorbs water easily, its exact concentration was hard to control in experiments. Therefore, in this study, the parameters, such as the solutal expansion coefficient $\left(\beta_{\mathrm{s}}\right)$, directly related to the concentration in the dimensionless groups are combined with the concentration (i.e., $C_{0} \beta_{\mathrm{s}}$ ) for best fitting the observed interface shapes. In this study, we do not attempt to perform an exact comparison. Therefore, the comparison, which is only qualitative, is used to understand the interplay physics. More quantitative comparison using acetone as the solute is under way. Detailed numerical implementation and parameter study, as well as quantitative comparison, are discussed elsewhere [14].

\section{Results and discussion}

Fig. 2 shows the interface evolution at three different rotation speeds. As shown by the case without rotation $(0 \mathrm{rpm})$, before solidification was started, the interface was very flat because the thermal conductivities of the melt and the crystal are very similar [3]. The ampoule translation speed was $2 \mu \mathrm{m} / \mathrm{s}$. After $1 \mathrm{~h}$, the interface became concave due to the release of heat fusion. Meanwhile, a clear depression or pit was formed at the interface center. This pit formation is due to solute accumulation, which lowers the liquidus temperature. A similar observation was reported by Schaefer and Coriell [3] for SCN/EtOH and by Singh et al. [15] for $\mathrm{PbBr}_{2} / \mathrm{AgBr}$. As the solidification proceeded further, more solute was accumulated in front of the interface, because the ethanol has a lower solubility in the solid than that in the melt $(k=0.044)$ [3]. Finally, as shown by the photograph at $100 \mathrm{~min}$, the freezing interface under the depression began to break down into a cellular structure. After $3 \mathrm{~h}$, one can see a clear trace left behind the depression.

Rotating at $100 \mathrm{rpm}$, the width of the depression was increased and the instability occurred much earlier (at about $50 \mathrm{~min}$ ). In fact, we believe that the Coriolis force partially balanced the gravitational forces and the convection was greatly suppressed. Because the transport of the solute into the bulk melt was reduced due to the weaker mixing, more solute accumulated near the solidification front. The flow direction near the solidification front remained the same. As a result, the depression remained at the interface center, but the solute accumulated faster. When the rotation rate was increased to $150 \mathrm{rpm}$, no depression was formed and there was no interface breakdown up to $3 \mathrm{~h}$. The interface shape before solidification started was more concave than in the other cases. As discussed shortly, this is attributed to forced convection caused by centrifugal acceleration.

When the rotating table was stopped at $3 \mathrm{~h}$, a spin-down flow (an inward flow near the interface) was observed, as shown by the last picture; some trajectories of tiny particles might help the observation in the photograph. This spin-down flow brought the solute from the periphery of the interface to the center and caused a sudden interface depression there. Apparently, at $150 \mathrm{rpm}$, the solute did not accumulate at the interface center and therefore the interface was 


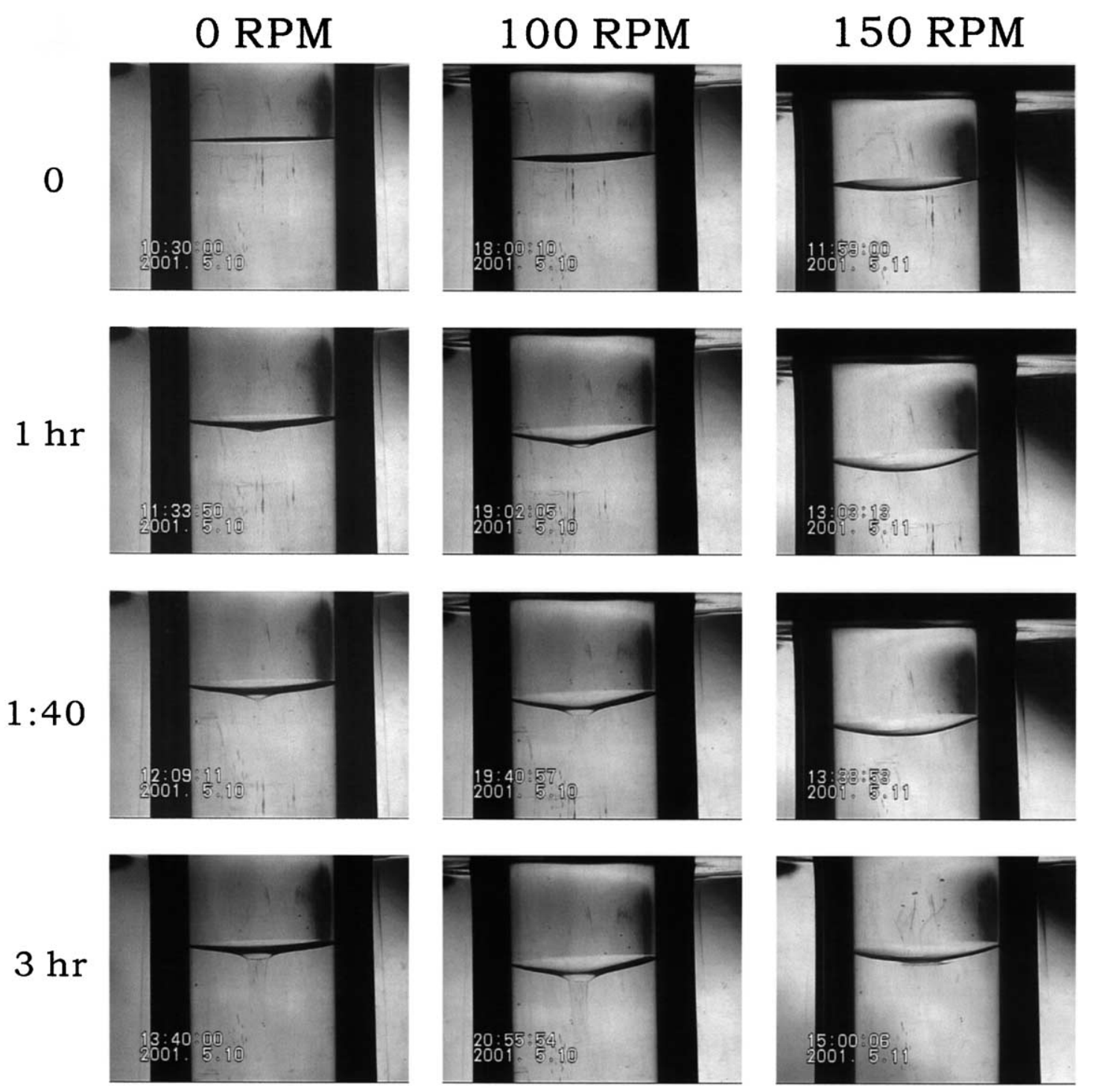

Fig. 2. Interface evolution at different rotation speeds.

stable. This also indicated that the radial solute segregation was reversed. Lan [11] also predicted, by numerical simulation, the inversion of radial gallium segregation for germanium growth.

A set of fully time-dependent simulations was also carried out to explain the observations. The results corresponding to the cases in Fig. 2 at $100 \mathrm{~min}$ are summarized in Fig. 3, but $85 \mathrm{rpm}$ was used for the second experiment. Because some physical properties, such as diffusivity and solutal expansion coefficients, are not available at this moment, we do not attempt to perform an exact comparison with the experiments. Nevertheless, the qualitative information from the simulation should be adequate for discussion. For the case of no rotation in Fig. 3a, an inward toroidal flow near the interface is predicted; in each figure, the solute field is on the right and the flow and thermal 


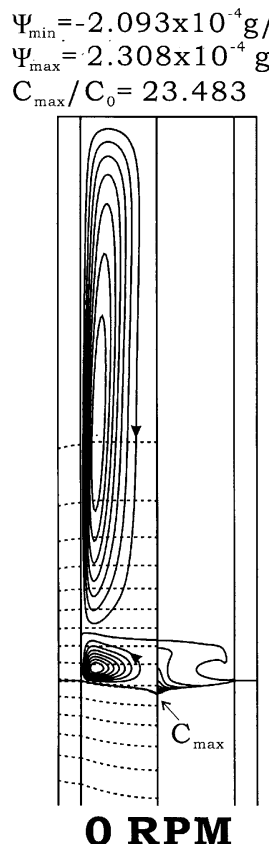

(a)

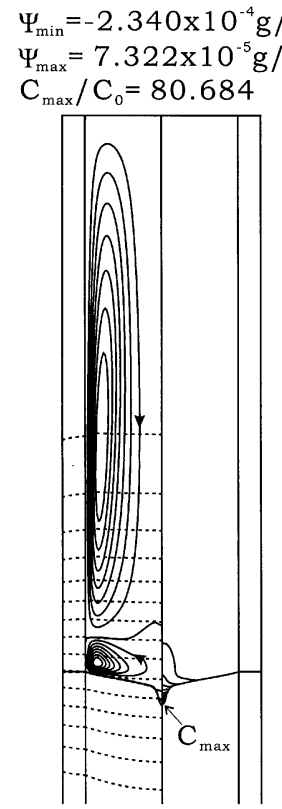

85 RPM

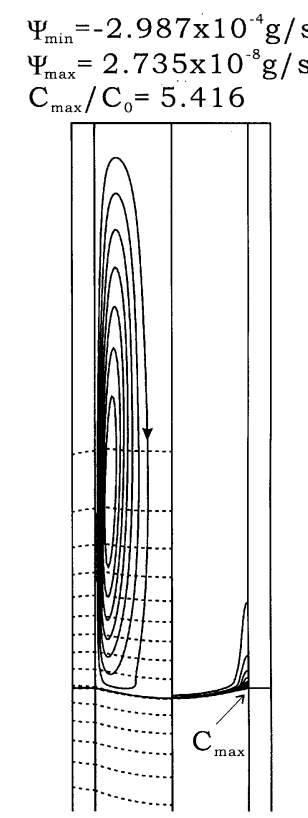

150 RPM

Fig. 3. Simulation results for Fig. 2 at $100 \mathrm{~min}$ : (a) $0 \mathrm{rpm}$; (b) $85 \mathrm{rpm}$; (c) $150 \mathrm{rpm}$; on the left-hand side of each figure is the contours of stream function $(\Psi)$ and isotherms (dashed line) and on the right-hand side of the normalized solute concentration $\left(C / C_{0}\right)$; $C_{0}$ is the initial solute concentration and the isotherm near the edge of the interface is the melting point of pure SCN.

(dashed line) fields on the left. This toroidal flow is caused by the concavity of the interface and is a result of both thermal and solutal effects. As discussed by Lan and $\mathrm{Tu}$ [4], at low solute concentration, thermal convection is the major driving force for the convection and is responsible for the pit formation. The major contribution of the solutal effect is near the center of the interface. Because ethanol is lighter than SCN, the flow near the depression, which contains more ethanol, is enhanced. The pit shape is affected significantly by the solutal effect [4]. The upper flow cell in the bulk melt is due to the thermal mismatch between the hot zone and the adiabatic zone [16], which is simulated by an ambient thermal profile having a hyperbolic tangent profile.

At $85 \mathrm{rpm}$, as shown in Fig. 3b, the flow is significantly suppressed (smaller stream function), and the solute is built up more quickly near the interface (larger $C_{\max } / C_{0}$ ). As a result, the depression is larger and deeper. At this rotation rate, the resultant gravitation acceleration is closer to the so-called magic- $g$ level [7-9], where the convection intensity is at the minimum. Finally, at $150 \mathrm{rpm}$, as shown in Fig. 3c, the flow structure near the interface is changed. The lower inward cell in front of the interface disappears leaving a big outward flow cell in the melt. As a result, the solute rejected from the interface is brought to the periphery of the interface. This flow is assisted by the centrifugal acceleration $\left(r \Omega^{2}\right.$, where $r$ is the radial coordinate and $\Omega$ the rotation speed), because the direction of centrifugal acceleration is perpendicular to the axial thermal gradient $(\mathrm{d} T / \mathrm{d} z)$. In fact, from the source term of the vorticity equation [14], the transition occurs at about $g \mathrm{~d} T / \mathrm{d} r \approx r \Omega^{2} \mathrm{~d} T / \mathrm{d} z$, where $g$ is the gravitational acceleration. Since the axial thermal gradient is about $10 \mathrm{~K} / \mathrm{cm}$ and the radial one $(\mathrm{d} T / \mathrm{d} r)$ about $1 \mathrm{~K} / \mathrm{cm}$, the critical rotation rate can be estimated as about $100 \mathrm{rpm}$, which is quite close to our experimental observations. 


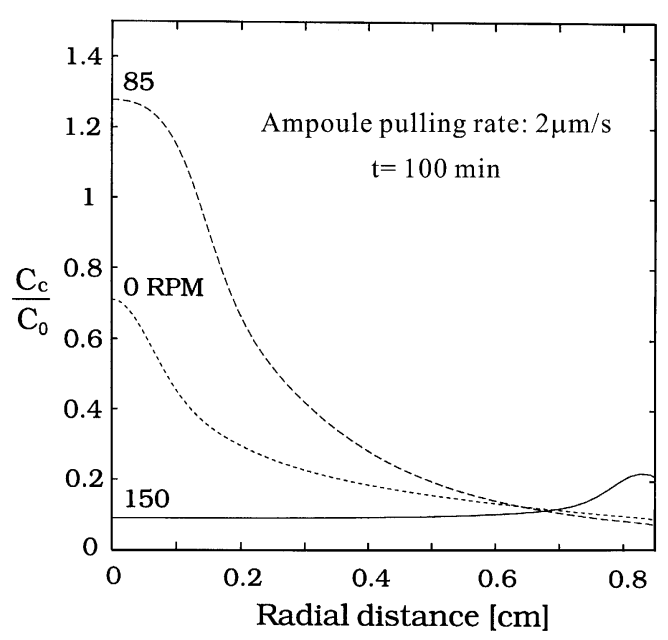

Fig. 4. Radial solute concentration at the interface at different rotation speeds ( $100 \mathrm{~min}$ after the ampoule translation).

In addition, because the periphery of the interface has a larger area to level down the solute concentration, the solute accumulation rate is much slower than that at the center. On the other hand, the center is a singular point having a small area to accommodate the solute and a faster build up of the solute is realizable. This can be better understood from the solute distributions at the interface shown in Fig. 4 (obtained from the results of Fig. 3). As shown, the accumulation of the solute at $150 \mathrm{rpm}$ at $100 \mathrm{~min}$ is one order smaller than that at $85 \mathrm{rpm}$. Furthermore, as shown in Fig. 3c, due to the enhanced flow by the centrifugal acceleration (one can compare the values of the stream function $\Psi_{\min }$ ), the isotherms can be more distorted by the flow leading to the more concave interface, even before the solidification starts. This was found in experiments (the first photograph in the third column of Fig. 2). One can also check the isotherms in Fig. 3c to get a better idea.

\section{Conclusions}

In this report, we present the observations of morphological evolution during vertical Bridgman growth of SCN containing ethanol in a rotational field. The rotation about the growth axis was found useful in convection and segregation control. Inversion of radial solute segregation and suppression of morphological instability due to pit formation can be achieved by rotation. Without rotation, the local solute accumulation at the interface center caused pit formation and destabilized the interface. At medium rotation speeds, where the minimum convection was found, the faster solute accumulation made the interface breakdown even earlier. However, once the rotation speed was high enough, the flow and thus the radial solute segregation near the growth front were reversed. The solute was brought to the periphery of the interface and accumulated less. As a result, the morphological stability was enhanced. Computer simulation was further carried out to explain the observation and good qualitative agreement was found.

\section{Acknowledgements}

This work was supported by the National Science Council of the Republic of China under Grant No. 89-2214-E-002-040. A careful review and comments by Prof. Ramasamy of Anna University are deeply appreciated.

\section{References}

[1] W.A. Tiller, K.A. Jackson, J.W. Rutter, B. Chalmers, Acta Metall. 1 (1953) 428.

[2] W.W. Mullins, R.F. Sekerka, J. Appl. Phys. 35 (1964) 444.

[3] R.J. Schaefer, S.R. Coriell, Metall. Trans. 15A (1984) 2109.

[4] C.W. Lan, C.Y. Tu, J. Crystal Growth 220 (2000) 619.

[5] H.A. Chedzey, D.T.J. Hurle, Nature 210 (1966) 933.

[6] A.F. Witt, H.C. Gatos, M.C. Lavine, C.J. Herman, J. Electrochem. Soc. 122 (1975) 296.

[7] H. Rodot, L.L. Regel, G.V. Sarafanov, H. Hamidi, I.V. Videskii, A.M. Turtchaninov, J. Crystal Growth 79 (1986) 77.

[8] J. Friedrich, J. Baumgartl, H.-J. Leister, G. Müller, J. Crystal Growth 167 (1996) 45.

[9] C.W. Lan, C.Y. Tu, J. Crystal Growth 226 (2001) 406.

[10] P.V. Skudarnov, L. Regel, W. Wilcox, J. Crystal Growth 193 (1998) 219.

[11] C.W. Lan, J. Crystal Growth 229 (2001) 595. 
[12] Y.W. Lee, R. Anauth, W.N. Gill, Chem. Eng. Commun. 152 (1996) 41.

[13] C.W. Lan, M.C. Liang, J. Crystal Growth 186 (1998) 187.

[14] C.W. Lan, H.Z. Chen, I.F. Lee, Morphological instability due to double diffusive flow during directional solidification in a rotational field, under preparation.
[15] N.B. Singh, S.S. Mani, J.D. Adam, S.R. Coriell, M.E. Glicksman, W.M.B. Duval, G.J. Santoro, R. DeWitt, J. Crystal Growth 166 (1996) 364.

[16] P.M. Adornato, R.A. Brown, J. Crystal Growth 80 (1987) 155. 